\title{
Optimizing Workflow in Electron Microscopes with Fast BSE/STEM Diodes and Preamplifier Modules
}

\author{
Maximilian Schmid ${ }^{1 *}$, Andreas Liebel ${ }^{1}$ and Heike Soltau ${ }^{1}$ \\ 1. PNDetector GmbH, Otto-Hahn-Ring 6, 81739 München, Germany \\ * Corresponding author: Maximilian.Schmid@pndetector.de
}

Modern electron microscopes more and more focus on fast sample processing from sample insertion to the final picture or information needed. From an economical view, this time bonus reduces machine time and staff costs. From the scientific view, the shorter the specimen is irradiated by electrons the less it can be influenced or damaged. Thus, an optimized workflow is a benefit for both worlds. However, not all types of detectors are able to support the capabilities offered by new Scanning Electron Microscopes (SEM) or (Scanning) Transmission Electron Microscopes ((S)TEM). In SEM Secondary Electron (SE) detectors are often used for coarse searching before switching to mostly slower Backscattered Electron (BSE) detectors. This costs time and leads to superfluous electron exposure to the specimen. Furthermore, the two different detector types provide different sample information, and thus hinder finding the region of interest on the specimen. Also in (S)TEM examinations fast diodes can support the rising scan speeds and facilitate the work with the microscopes. Here, we show how fast diodes and electron detector modules can help to achieve this goal.

Fig. 1 displays a BSE detector module where diode and preamplifier board form one unit, placed in a flat high-grade housing, to support the high speeds of the BSE-Chip. The closest connection of the BSEDiode to the preamplifier board ensures minimum stray capacitance between signal generation and amplification. In addition, the design of the amplifier is geared to the diode, which enables the BSE detector rise times down to $20 \mathrm{~ns}$, at an amplification factor of $10^{5} \mathrm{~V} / \mathrm{A}$. Fig. 2 shows a single frame from a full HD video (1920x1080 pixel) captured at a frame rate of $24 \mathrm{~Hz}$ highlighting the outstanding speed of the BSE detector module. TV speed imaging at high resolutions helps to search and identify sample regions as well as acquiring high quality pictures without the need for a search in reduced image resolution mode and ensures a minimal interaction time between the electron beam and the specimen.

Next to the high speeds the diodes are capable to work at low signal conditions. A standard diode with $80 \mathrm{~mm}^{2}$ sensitive area exhibits a maximum Geometric Collection Efficiency (GCE) of 55\% at a distance of $3 \mathrm{~mm}$ between the sample and diode. Such high GCE enable high signal outputs even at low beam energies and low beam currents. Fig. 3 shows a comparison of BSE images taken at various beam energies from $20 \mathrm{keV}$ down to $1 \mathrm{keV}$. The series indicates the different information when altering the interaction volume by adjusting the electron energy. While at $20 \mathrm{keV}$, areas of different carbon coating thickness cannot be detected, the darker and lighter domains indicate the surface sensitivity at 3 and $1 \mathrm{keV}$.

This talk will show the possibilities and benefits of high speed detectors in a variety of applications in material and life science in SEM as well as in (S)TEM. It will take into account high throughput applications as well as work with beam sensitive specimen. 


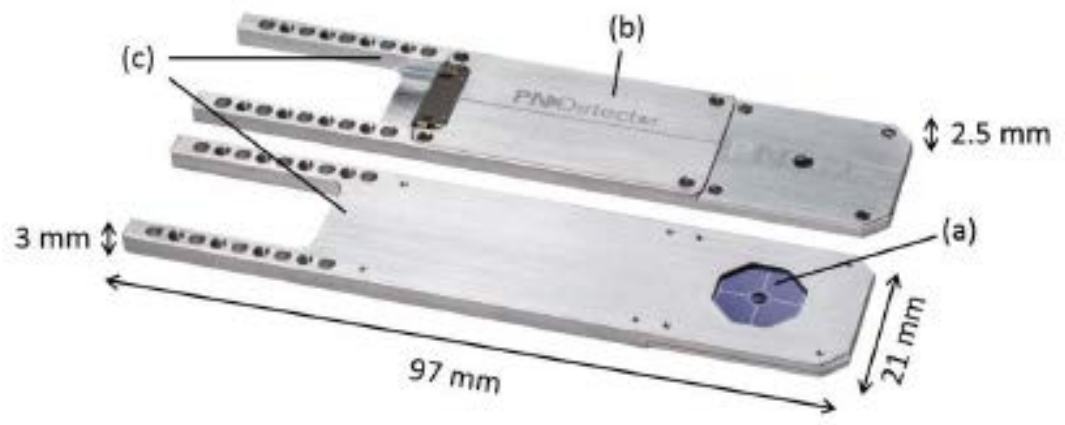

Figure 1. Front and backside view of the high speed BSE detector module: (a) 4 Quadrant BSE Diode, (b) 4 channel preamplifier, directly connected to the diode, (c) ultra flat aluminum housing to fit below the pole piece.

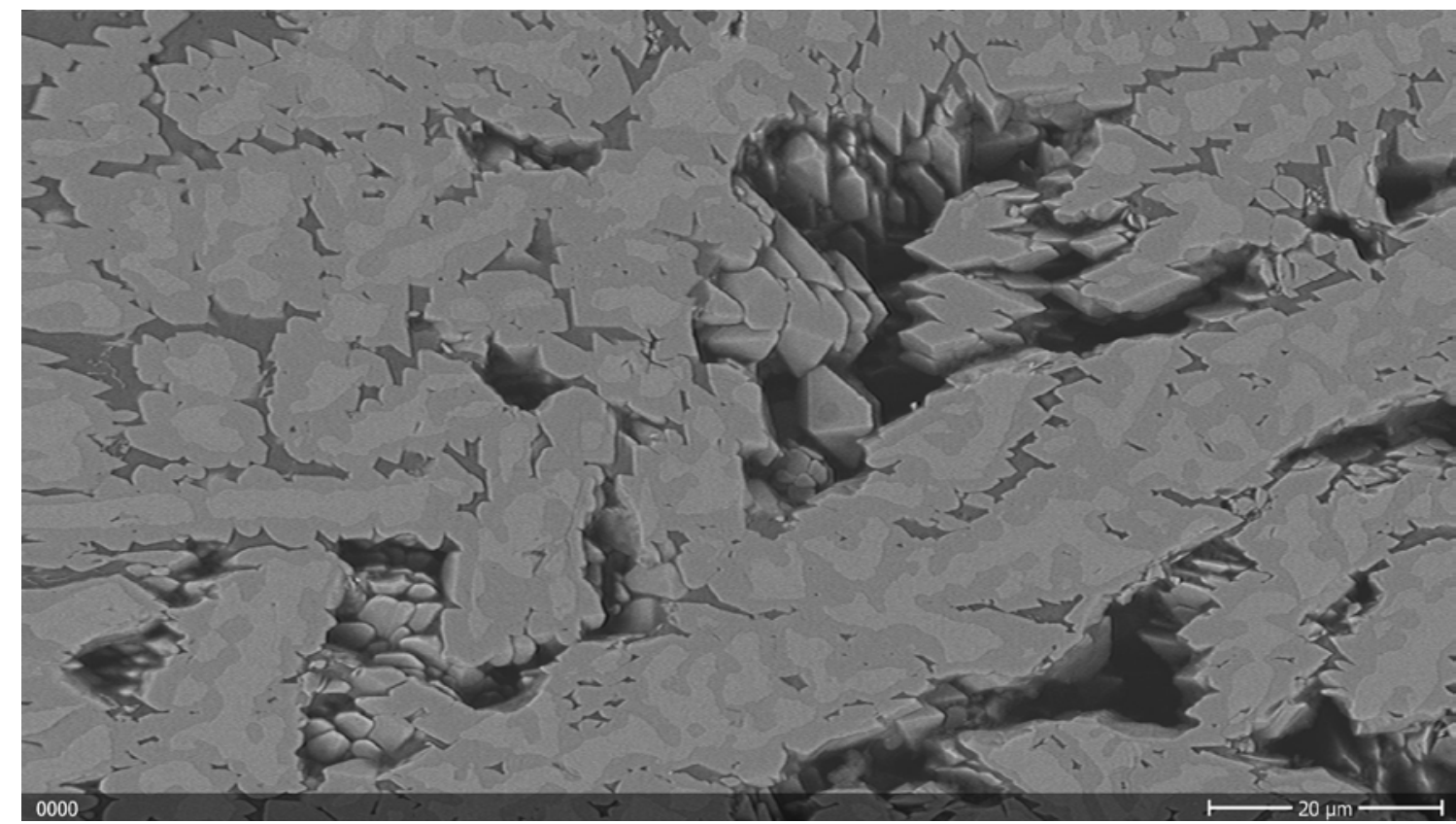

Figure 2. Single frame of a full HD movie of a Raney Nickel sample recorded live in SEM. Movie recorderd with: $E_{\text {beam }}=20 \mathrm{keV}$, Ibeam $=800$ pA, 1920*1080 pixel, $t_{d w e l l}=20 \mathrm{~ns}, 24 \mathrm{fps}$.
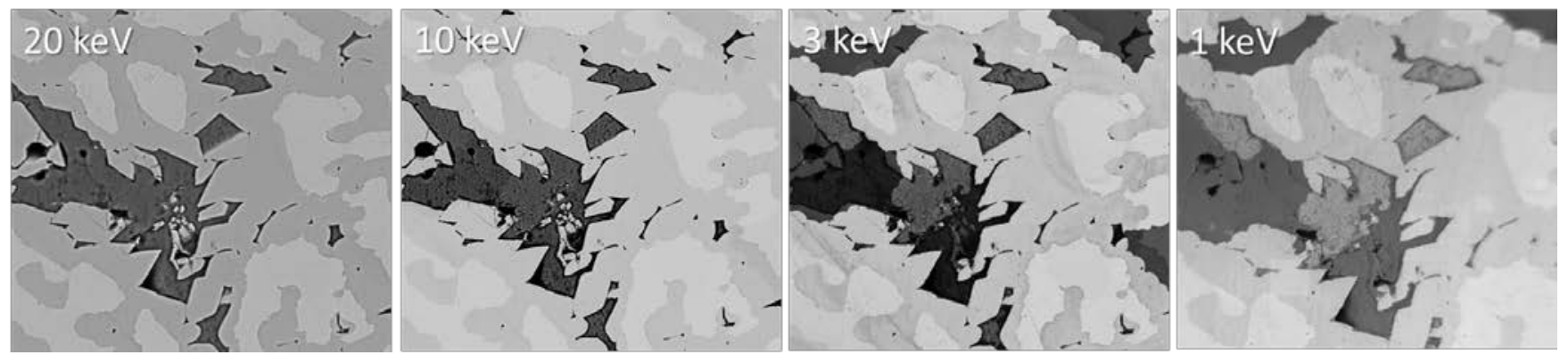

Figure 3. Comparison of a Raney Nickel specimen at beam energies ranging from $\mathrm{E}_{\text {beam }}=20 \mathrm{keV}$ to $\mathrm{E}_{\text {beam }}=1 \mathrm{keV}$. The highlighted area shows the different contrast originating from the shrinking interaction volume of the electron beam with the sample for lower energies. 